\title{
GEOMORFOLOGÍA Y CARTOGRAFÍA DEL SECTOR CENTRAL DE CARTAGENA DE INDIAS - COLOMBIA
}

\section{GEOMORPHOLOGY AND MAPPING OF THE CENTRAL SECTOR OF CARTAGENA DE ÍNDIAS - COLOMBIA}

\author{
Max Furrier ${ }^{1}$ \\ Universidade Federal da Paraíba, Brasil \\ German Vargas Cuervo ${ }^{2}$ \\ Universidad Nacional de Colombia, Bogotá
}

\begin{abstract}
RESUMEN
El presente trabajo se refiere a la caracterización geomorfológica, morfoestructural y morfométrica de la región inmersa en la hoja 23 III D, localizada al Noreste de Colombia. La utilización de las técnicas de geoprocesamiento para la evaluación geomorfológica y morfoestructural, además de intentar reforzar las hipótesis levantadas en estudios anteriores, es considerada relevante, pues los productos generados son notables para así evidenciar de manera concisa la morfología del relieve, donde la influencia morfoestructural y tectónica en su configuración y disposición es demasiado fuerte. Las técnicas utilizadas en esta investigación consistirán en la elaboración y análisis de las planchas hipsométrica, de inclinación de las pendientes y geomorfológica, perfiles topográficos y cálculos morfométricos. Los resultados obtenidos a partir de los análisis de los productos generados muestran que la configuración del relieve está vinculada a una fuerte influencia morfoestructural y tectónica, incompatibles con una evolución basada única y exclusivamente en los aspectos climáticos, además de una intensa erosión costera de influencia antrópica.
\end{abstract}

Palabras clave: Erosión Costera. Análisis Morfoestructural. Sistema de Informaciones Geográficas. Morfometría.

1 Doutor em Geografia Física, Departamento de Geociências, Universidade Federal da Paraíba, Brasil. Correo electrónico: max.furrier@gmail.com

2 Doctor en Ciencias de la Tierra, Departamento de Geografía, Universidad Nacional de Colombia, Bogotá. Investigador Junior (IJ). Correo electrónico: gvargasc@unal.edu.co

Fecha de recepción: 16 de marzo de 2016

Fecha de aceptación: 8 de mayo de 2016 
Max Furrier, German Vargas Cuervo. Geomorphology and mapping of the Central Sector of Cartagena de Índias - Colombia

\begin{abstract}
This study refers to the geomorphological, morphostructural and morphometric characterization of the onshore region of 23 III D chart, located in the Northeast of Colombia. Besides aiming at reinforcing the hypothesis raised by previous studies, the use of GIS techniques for geomorphological and morphostructural evaluation is considered relevant because the products generated are remarkable to concisely highlight the morphology of the relief, where the morphostructural and tectonic influence in its configuration and arrangement is conspicuous. The techniques used in this study consisted of the preparation and analysis of the hypsometric and declivity charts, as well as the analysis of topographic profiles generated in this study. The results obtained from the analysis of the products showed that the configuration of the relief was linked to a strong morphostructural and tectonic influence, incompatible with an evolution based solely on climatic aspects, in addition to an intense coastal erosion of human influence.
\end{abstract}

Keywords: Coastal Erosion. Morphostructural Analysis. Geographic Information System. Morphometric.

\title{
Introducción
}

En virtud de la complejidad natural y del nivel de intervención humana en la organización del espacio geográfico del ambiente costero, este segmento del relieve viene ganando atención cada vez más grande en cuanto a la manutención de su equilibrio, lo que conlleva por tanto a la necesidad de obtener un conocimiento detallado de sus estructuras y de las fuerzas que intervienen en la adaptación de sus formas (Feitosa, 1996).

En efecto, las formas del relieve son estructuras dinámicas y heterogéneas que se modifican en el tiempo y espacio de acuerdo con la presencia y la intensidad de los elementos que las originan y/o las extinguen, siendo estos de naturaleza endógena, climática o antrópica. Los procesos de naturaleza endógena y climática son objeto de estudio de la geomorfología, los cuales son históricamente concebidos y aceptados. En consecuencia, no se puede comprender una Geomorfología superficial desconsiderando el sustrato geológico y basamento rocoso.

Diversas entidades han utilizado tangencialmente la información geomorfológica con diferentes propósitos, en algunos casos únicamente con el objeto de cumplir un requisito de los informes geológicos y de ingeniería. No obstante lo anterior, la necesidad de llevar a cabo tanto planes de planificación ambiental, el ordenamiento territorial y de conocer la estructura del relieve, y no solamente sus formas superficiales, ha permitido vislumbrar el valor que tiene la geomorfología, no solo por la información que proporciona, sino también porque facilita la tarea de definir zonas morfoestructurales homogéneas y su mapeo (Carvajal, 2012). 
A través de este trabajo, se pretende contribuir para un mejor conocimiento de la dinámica de los procesos geomorfológicos y estructurales; así como de su relación con la actual configuración del relieve en la porción central de la ciudad de Cartagena de Indias y alrededores, contribuyendo así a enriquecer los datos actualmente disponibles, lo cual podrá servir de ayuda tanto para futuros proyectos de planificación ambiental y territorial, como para futuras investigaciones que se desarrollen en esta fracción de la ciudad que es de suma relevancia en términos turísticos para Colombia.

\section{Área de estudio}

El área de estudio se encuentra entre las coordenadas 1'650.000 N, 1'640.000 S, $850.000 \mathrm{~W}$ y $836.000 \mathrm{E}$, comprendiendo la hoja $23 \mathrm{III}-\mathrm{D}$ (IGAC, 1996), encontrándose en el sector central de la jurisdicción de la ciudad de Cartagena de Indias - Departamento de Bolívar, en la región del caribe colombiano.

El casco urbano de la ciudad se encuentra ubicado alrededor de la Ciénaga la Virgen. El área posee un clima tropical seco con temperatura media de $29^{\circ} \mathrm{C}$, con algunas variaciones locales y lluvias anuales de 900 $\mathrm{mm}$. Los vientos más fuertes se presentan de diciembre a abril debido a la influencia de los alisios del norte (INGEOMINAS, 2004). 
Max Furrier, German Vargas Cuervo. Geomorphology and mapping of the Central Sector of Cartagena de Índias - Colombia

Figura 1. Localización del área de estudio y sus respectivas formaciones geológicas

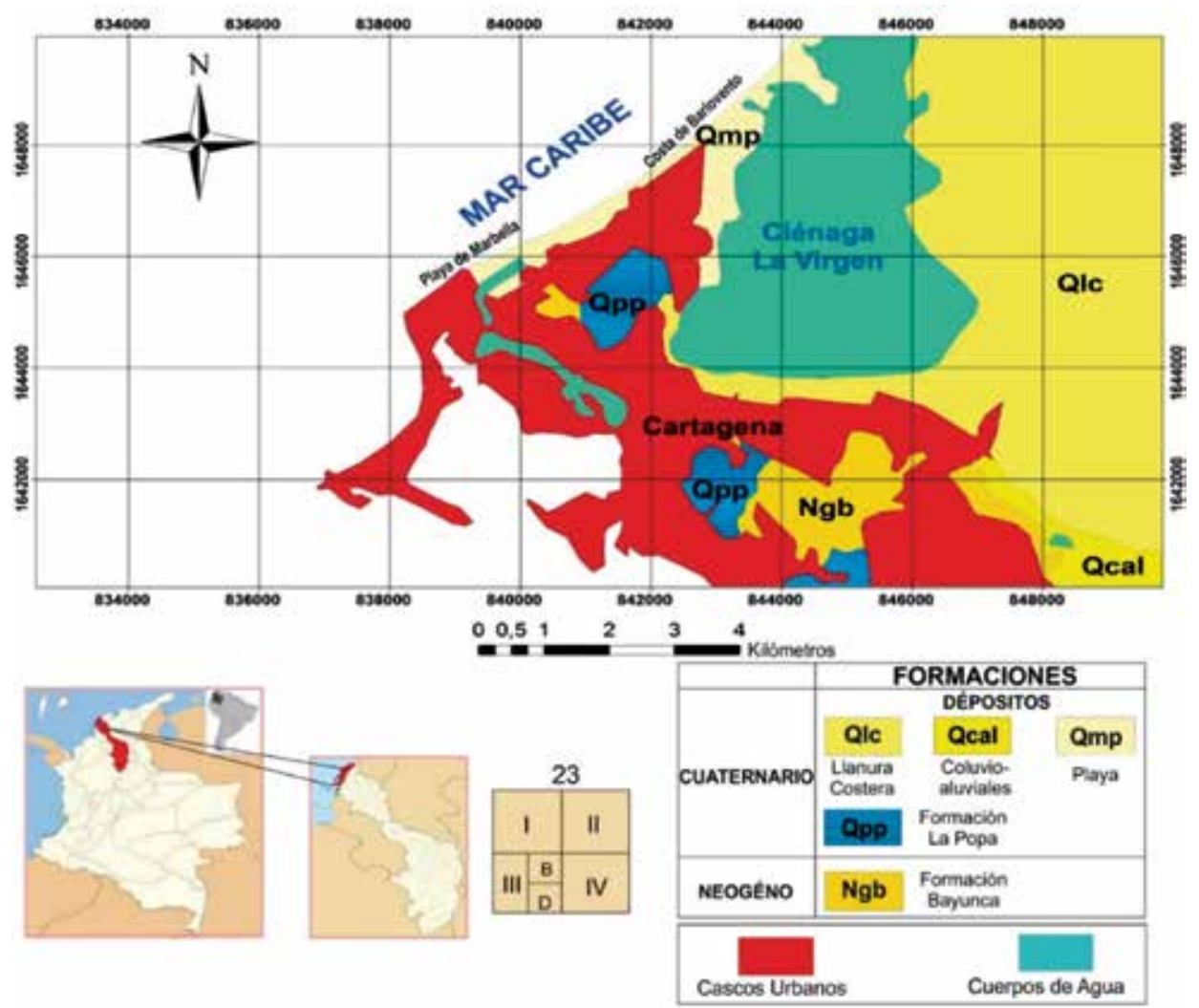

Fuente: Adaptado de IGEOMINAS, 2004.

El caribe colombiano, se encuentra situado precisamente en la convergencia entre las placas del Caribe y de América del Sur; por tanto, constituye un punto clave para entender la evolución geomorfológica en márgenes continentales activos. Es importante tener en cuenta que las formaciones Bayunca y La Popa, descritas en este trabajo, fueron depositadas en el lecho marino somero en un margen continental activo y que, debido a la intensa compresión y flexión, actualmente, se encuentran en la superficie formando parte importante del relieve actual (INGEOMINAS, 2004). 


\section{Características geológicas y geomorfológicas generales}

La ciudad de Cartagena se encuentra predominantemente ubicada sobre sedimentos cuaternarios, compuestos por depósitos de llanura costera y depósitos de playa; además, se encuentran afloramientos de la Formación La Popa que reposa de forma discordante sobre la Formación Bayunca del Plioceno (Figura 1).

La Formación Bayunca tiene origen en una zona entre costa afuera y plataforma continental, luego sus características de sedimentación muestran también sedimentos formados en una zona intermareal, la cual es a su vez afectada por canales con abundante concentración de conchas y en otras partes areniscas rellenando canales abandonados con contactos erosivos. En detalle, se observa también estratificación ondulada paralela que puede ser completamente borrada por la alta bioturbación.

Suprayaciendo la Formación Bayunca de forma discordante se encuentra la Formación La Popa del Pleistoceno Superior. Angel et al (1985) utilizan el término Formación La Popa, para describir rocas expuestas en el Cerro La Popa en Cartagena. Particularmente, estos autores, para referirse al miembro inferior utilizaran el término "unidad detrítica de La Popa" y para el superior, "calizas arrecifales de La Popa".

Para la Formación La Popa se sugiere arrecifes formados en una plataforma submarina con una pendiente muy suave y por debajo del nivel de base de las olas, en un área con poco aporte de sedimentos, aguas claras y temperaturas entre $21^{\circ}$ y $25^{\circ} \mathrm{C}$ que permiten el desarrollo de estos cuerpos calcáreos (INGEOMINAS, 2004).

Los depósitos de llanura costera (Qlc) forman áreas planas al nordeste del casco urbano de Cartagena y gran parte de sus cursos de agua convergen en la Ciénaga de la Virgen. Dichos depósitos presentan un régimen de depósito progradante, lo cual conlleva la formación de depósitos orgánicos y clásticos. En cuanto a los depósitos orgánicos son principalmente los manglares; mientras que en los depósitos clásticos se consideran las arenas y arcillas depositadas y retrabajadas.

Los depósitos de playas (Qmp) están localizados a lo largo de la línea de costa y constan de arenas de grano fino a grueso, ocasionalmente con gravas; la mayoría es de color amarillo ocre a gris. Estos depósitos poseen un alto contenido de carbonato, lo cual fue comprobado por la reacción con ácido de cloruro. Su área geográfica de mayor exposición es 
precisamente la línea de costa del área objeto de estudio, la cual se encuentra densamente ocupada por una urbanización desproveída de cualquier tipo de planificación u ordenamiento territorial.

En menor proporción se encuentran los depósitos coluvio-aluviales (Qcal) que agrupan tanto la sedimentación fluvial reciente y actual, como la generada por coluviones igualmente recientes, relacionados con arroyos y piedemontes. Tales depósitos coluvio-aluviales están constituidos por cantos, bloques, arenas, arcillas y mezclados agradados, los cuales cubren las unidades rocosas y los depósitos cuaternarios más antiguos.

\section{Marco teórico conceptual de la Cartografía Geomorfológica utilizada}

La fundamentación teórico-metodológica para la elaboración de la plancha geomorfológica propuesta para este trabajo es la desarrollada por Ross (1992), que constituye una evolución de los conceptos presentados por el geógrafo soviético Mescerjakov (1968) y de los conceptos y técnicas desarrollados en el Proyecto RADAMBRASIL (Barbosa et al., 1984), con ajustes propuestos por Furrier (2007) para la adaptación de la escala utilizada en este trabajo. La metodología de Ross (op. cit) establece que el relieve está dividido por taxones jerárquicos, organizados y descritos conforme el cuadro 1 y la figura 2 . 


\section{Cuadro 1. Representación esquemática de las unidades taxonómicas propuestas por Ross (1992)}

\begin{tabular}{|l|l|}
\hline $1^{\text {er }}$ Taxón & $\begin{array}{l}\text { Unidad morfoestructural - son las estructuras geológicas más amplias, } \\
\text { como las cadenas de montañas, macizos, mestas y depresiones internas } \\
\text { de los continentes y de los océanos. }\end{array}$ \\
\hline $2^{\text {do }}$ Taxón & $\begin{array}{l}\text { Unidades morfoesculturales - se relacionan con la acción de los } \\
\text { elementos morfogenéticos sobre las morfoestructuras. Son las formas } \\
\text { resultantes. Pueden ser caracterizados como rasgos del relieve generado } \\
\text { sobre las morfoestructuras a través de procesos exógenos y endógenos. } \\
\text { Son morfoesculturas las mesetas, sierras, planicies entre otros. }\end{array}$ \\
\hline $3^{\text {er }}$ Taxón & $\begin{array}{l}\text { Patrones de formas del relieve - este taxón se refiere a una determinada } \\
\text { unidad morfoescultural de dimensión inferior, siendo en este taxón que } \\
\text { los procesos morfoclimáticos actuales comienzan a ser percibidos. Los } \\
\text { patrones de formas del relieve pueden ser: formas de acumulación, } \\
\text { como planicies fluviales y marinas, o formas propias de procesos } \\
\text { denudacionales, como colinas, cerros mesetas, entre otros. }\end{array}$ \\
\hline $4^{\text {to }}$ Taxón & $\begin{array}{l}\text { Tipos de forma del relieve - este taxón está fundamentado en la } \\
\text { disección del relieve. El mapeo lleva en consideración las dimensiones } \\
\text { interfluviales y el grado de profundidad del drenaje. En el relieve } \\
\text { en meseta prevalece la pedogénesis, considerando la superioridad } \\
\text { de la infiltración sobre el escurrimiento. El grado de evolución del } \\
\text { relieve refleja en conjunto los componentes tratados (pedogénesis y } \\
\text { morfogénesis), que puede ser justificado por la intensidad de incisión } \\
\text { del drenaje, cuya densidad se refleja en la dimensión interfluvial. En } \\
\text { resumen, es la morfometría del relieve. }\end{array}$ \\
\hline $5^{\text {to }}$ Taxón & $\begin{array}{l}\text { Sectores de las pendientes - pertenecen a cada una de las formas } \\
\text { individualizadas del relieve, cuya forma puede ser convexa, rectilínea } \\
\text { y cóncava. }\end{array}$ \\
$\begin{array}{l}\text { Pequeñas formas del relieve - aquellas resultantes de procesos actuales; } \\
\text { por ejemplo, cárcavas, hondonadas y bancos de sedimentación, además } \\
\text { de formas producidas por el hombre como cortes y rellanos entre otros. }\end{array}$ \\
\hline
\end{tabular}


Max Furrier, German Vargas Cuervo. Geomorphology and mapping of the Central Sector of Cartagena de Índias - Colombia

Figura 2. Representación esquemática de las unidades taxonómicas

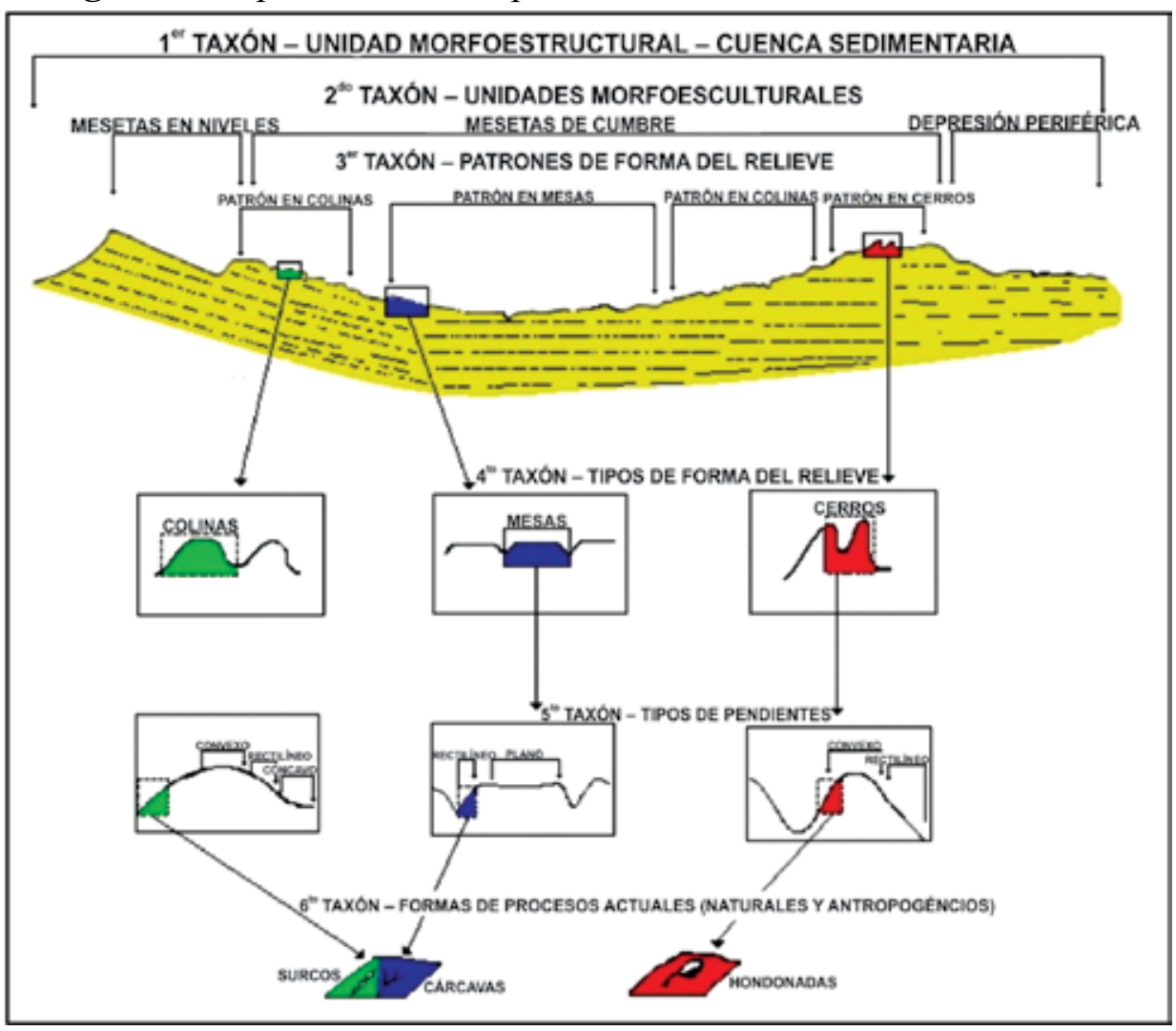

Fuente: Modificada de Ross, 1992.

\section{Marco metodológico}

Los procesos metodológicos propuestos en este trabajo fueron la revisión bibliográfica referente a la geología y los rasgos geomorfológicos generales enfocados al área de estudio; la consulta de materiales cartográficos, fotografías aéreas e imágenes de satélite para apoyar la interpretación del terreno; la elaboración de planchas temáticas de hipsometría y de inclinación de las pendientes que sirvieron de ayuda tanto para la lectura como para la elaboración de la plancha geomorfológica y; por último, la elaboración de la plancha geomorfológica del área de estudio. 


\section{Elaboración de las planchas de hipsometría e inclinación de las pendientes}

Inicialmente, fue vectorizada y digitalizada la hoja topográfica 23 III D de escala 1:25.000 con curvas de nivel a equidistancia de 25 metros; seguidamente, fueron generadas las matrices regular e irregular a partir de una imagen SRTM con resolución de $30 \mathrm{~m}$ y; finalmente, fueron generadas curvas de nivel con equidistancia de $1 \mathrm{~m}$ y un MNT (Modelo Numérico del Terreno), todo lo anterior a fin de elaborar las planchas de hipsometría e inclinación de las pendientes. Cabe resaltar que para ésta última se utilizó la clasificación propuesta por Herz y De Biase (1989) muy utilizada en Brasil, país que constituye una referencia para los estudios realizados en ese campo en Sur América. El SIG utilizado fue el software ArcGis ${ }^{\circledR}$ v 10.2.

Para la elaboración de la plancha de hipsometría, fue utilizada la matriz regular y; posteriormente, su reclasificación en clases de altimetría en intervalos de $0-5,5-20,20-40,40-60,60-80,80-100,100-120$ $\mathrm{y}>120 \mathrm{~m}$. con los respectivos colores que van de verde en las zonas más bajas a rojo en las zonas más altas.

Para la elaboración de la plancha de inclinación de pendientes, se utilizó la matriz regular y se crearon las clases de pendientes, que varían de 0 a $>100 \%$, atribuyendo sus debidos colores. La definición de las clases de inclinación de las pendientes usadas en este trabajo fue ajustada a las clases de límites utilizados internacionalmente y al Código Florestal de Brasil, que presenta una clasificación útil para el abordaje analítico adoptado en el presente estudio. Esas clases fueron catalogadas de la siguiente forma:

- $\quad<12 \%$ : el límite máximo de empleo de mecanización en la agricultura;

- $\quad 12-30 \%$ : limita a 30\% la inclinación de la pendiente para urbanización sin restricciones;

- $\quad 30-47 \%$ : limita a $47 \%$ la inclinación de las pendientes en las que es posible realizar la tala de vegetación;

- $\quad 47-100 \%$ : en este intervalo de inclinación de las pendientes el Código Florestal de Brasil prohíbe la tala de bosques sin un régimen de utilización racional que sea destinado a ingresos permanentes;

- $\quad>100 \%$ : es considerada, por el Código Florestal de Brasil área de preservación permanente, apenas siendo admitida la eliminación total o parcial de la vegetación, con previa autorización de las autoridades 
Max Furrier, German Vargas Cuervo. Geomorphology and mapping of the Central Sector of Cartagena de Índias - Colombia

públicas, cuando fuera necesaria la ejecución de actividades o proyectos de utilidad pública o de interés social.

\section{Elaboración de la plancha geomorfológica}

Para la elaboración de la plancha geomorfológica producto de esta investigación, fue necesaria la superposición e interpretación de los productos cartográficos ya mencionados y además de esto, trabajos de campo. Para un mejor entendimiento todo el proceso será descrito en 5 etapas:

Etapa 1: determinación del primer taxón referente a la morfoestructura. En el área de estudio fueron identificados cuatro dominios: Depósitos de Playa, Depósitos de Llanura Costera, Depósitos Coluvio-aluviales y el Cinturón del Sinú (Cinturón de Plegamiento).

Etapa 2: determinación de la morfoescultura; es decir, las grandes formas del relieve predominantes en el área. Para el sector central de Cartagena fueron identificadas cuatro: Terrazas y Planicies Marinas, Terrazas y Planicies de Costeras, Terrazas y Planicies coluvio-aluviales.

Etapa 3: determinación de los patrones de forma del relieve. Para la determinación de este taxón fue necesario el uso de una imagen sombreada, una imagen de satélite y de la hoja topográfica, a fin de poder evidenciar los patrones de formas del relieve, desde formas de denudación hasta de acumulación.

Etapa 4: determinación de los tipos de formas del relieve. Para esta investigación fueron clasificadas tres formas de acumulación; a saber: (formas de terraza y planicies marina, formas de terrazas y planicies fluviales y formas de terraza y planicies coluvio-aluviales). Las formas de denudación en el presente trabajo se dividen en dos tipos: formas en meseta y convexa. Dentro de estos tipos a su vez, en esta división existen subclasificaciones que se determinan mediante el siguiente procedimiento: se traza una recta en la región central del curso principal de una determinada cuenca; y en seguida, se observa la diferencia altimétrica entre las dos extremidades de la recta. En las columnas de la tabla 1 se encuentra la clase de entallamiento (incisión) medio de los valles que va de muy débil hasta muy fuerte. En las filas de la misma se mide la longitud de los divisores de agua, donde se encuentra el curso de agua, que dice respecto a la dimensión interfluvial media, que va desde muy grande a muy pequeña (Tabla 1). 
Tabla 1. Matriz de los índices de disección de las formas del relieve

\begin{tabular}{|l|c|c|c|c|c|}
\hline \multirow{2}{*}{$\begin{array}{c}\text { Grado de } \\
\text { Entallamiento } \\
\text { de los Valles }\end{array}$} & \multicolumn{5}{|c|}{ Dimensión Interfluvial Media } \\
\cline { 2 - 6 } & $\begin{array}{c}\text { Muy } \\
\text { Grande (1) } \\
\mathbf{1 5 0 0} \mathbf{~ m}\end{array}$ & $\begin{array}{c}\text { Grande (2) } \\
\mathbf{1 5 0 0} \text { a 700 m }\end{array}$ & $\begin{array}{c}\text { Media (3) } \\
\mathbf{7 0 0} \text { a 300 } \mathbf{~ m}\end{array}$ & $\begin{array}{c}\text { Pequeña (4) } \\
\mathbf{3 0 0} \text { a 100 } \mathbf{~ m}\end{array}$ & $\begin{array}{c}\text { Muy } \\
\text { Pequeña (5) } \\
\leq \mathbf{1 0 0} \mathbf{~ m}\end{array}$ \\
\hline Muy débil (1) $<10 \mathrm{~m}$ & 11 & 12 & 13 & 14 & 15 \\
\hline Débil (2) $10-20 \mathrm{~m}$ & 21 & 22 & 23 & 24 & 25 \\
\hline Medio (3) $20-40 \mathrm{~m}$ & 31 & 32 & 33 & 34 & 35 \\
\hline Fuerte (4) $40-80 \mathrm{~m}$ & 41 & 42 & 43 & 44 & 45 \\
\hline Muy fuerte (5) $>80 \mathrm{~m}$ & 51 & 52 & 53 & 54 & 55 \\
\hline
\end{tabular}

Fuente: Adaptada de Ross (1992)

En el presente trabajo fueron clasificadas, en general, dos formas denudacionales (formas en meseta 22 y formas convexa 42 ).

Etapa 5: la identificación del quinto taxón consistió en un análisis de las pendientes observadas en campo tomando en consideración la plancha de inclinación de las pendientes, el perfil topográfico producto de esta investigación y la erosión costera que domina las playas urbanas de la ciudad. La descripción de estas pendientes no estará en la plancha geomorfológica debido a su escala, mas será incorporada en el texto como una figura.

Etapa 6: El sexto taxón consiste en las características naturales actuales identificadas. Par el caso concreto el análisis se fundamentó en una imagen de satélite, fotografías aéreas e información recolectada en trabajos de campo, donde las formas fueron verificadas y luego delineadas con el auxilio de un GPS.

\section{Resultados}

La elaboración de la plancha hipsométrica del área de estudio, permitió realizar el análisis de la variación topográfica, identificando alturas entre los $0-120$ metros en relación con el nivel del mar. Se verifica, que la distribución de las cotas más elevadas no se da de forma gradual, principalmente, en el Cerro La Popa, en el sector occidental del área (Figura 3).

La Formación Bayunca, del Neógeno posee elevaciones máximas de $40 \mathrm{~m}$, formando un relieve en mesetas. La formación que presenta mayor heterogeneidad es la Formación La Popa del Pleistoceno tardío que es la más reciente formación del Cinturón del Sinú y que presenta altitudes más 
discrepantes formando muchas veces pequeños cerros con formas variadas, existiendo inclusive un cerro en el casco urbano de Cartagena de Indias que llega a tener una altitud de 120 metros por el (MNT); no obstante, lo cual a partir de la información obtenida en los trabajos de campo en consonancia con puntos tomados con GPS se deduce que la altura máxima es de $146 \mathrm{~m}$.

Las altitudes y formas verificadas en la Formación La Popa, constituida por calizas arrecifales, solo pueden ser explicadas por actividad tectónica pos-pleistoceno de intensidad considerable. En efecto, teniendo en cuenta que las tasas de meteorización de calizas son altas para el tipo de clima encontrado en el área, y que en dicha área no existe una tectónica reciente de actividad moderada a fuerte, no cabe la posibilidad de encontrar esta formación en las altitudes verificadas por razones distintas de la actividad tectónica pos-pleistoceno. En cuanto a la acción tectónica reciente es importante destacar que la misma se evidencia en las fallas geológicas verificadas en las exposiciones mayores de esta formación.

Figura 3 - Plancha hipsométrica del área de estudio

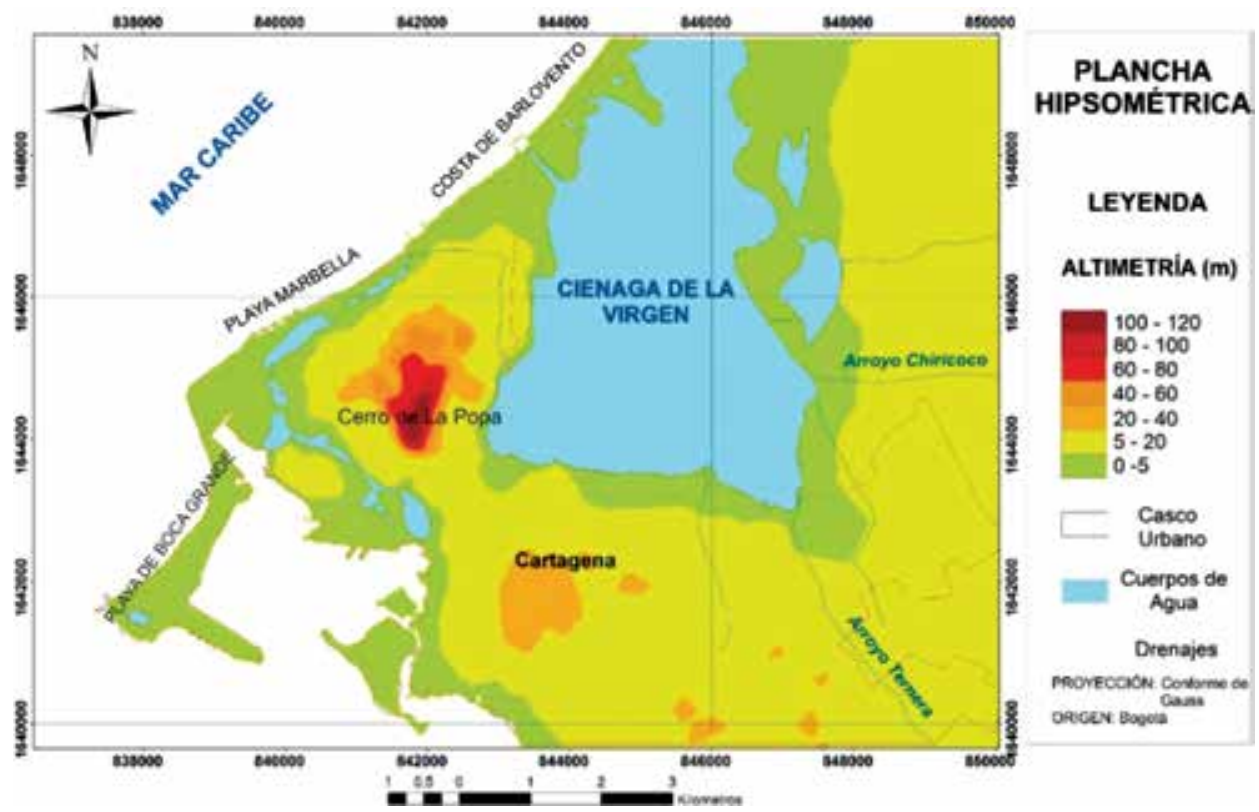


Los depósitos cuaternarios presentan las altitudes más bajas, casi siempre debajo de $20 \mathrm{~m}$ y con predominancia altimétrica a niveles debajo de $10 \mathrm{~m}$. Estos depósitos cuaternarios abarcan la mayor área geográfica de la plancha 23 III D. Los depósitos de playas forman terrazas y planicies marinas y nunca pasan la cota de $5 \mathrm{~m}$ de altitud.

La tabla 2 muestra la proporción en $\mathrm{km}^{2}$ y en porcentaje de cada clase hipsométrica del área de estudio.

Tabla2. Clases de hipsometría en el área de estudio en $\mathrm{km}^{2}$ y en porcentaje

\begin{tabular}{|c|c|c|}
\hline Clases & Área (km2) & Área (\%) \\
\hline $0-5$ & 40,32 & 45,91 \\
\hline $0-20$ & 43,25 & 49,24 \\
\hline $20-40$ & 2,89 & 3,29 \\
\hline $40-60$ & 0,66 & 0,75 \\
\hline $60-80$ & 0,37 & 0,43 \\
\hline $80-100$ & 0,17 & 0,19 \\
\hline $100-120$ & 0,16 & 0,18 \\
\hline
\end{tabular}

La plancha de inclinación de las pendientes muestra la declividad del terreno, que va de $0-100 \%$, donde se identifica una topografía relativamente plana, con un amplio predominio de valores de inclinación de las pendientes de $0-12 \%$, como se puede observar en la plancha de inclinación de las pendientes (Figura 4 ). Las pendientes $<3 \%$ son verificadas en los depósitos sedimentarios cuaternarios, pues los mismos son productos de deposición actual y continúa, debido a que esas áreas serán constantemente sometidas a inundaciones por sus bajas altitudes. Estas inundaciones frecuentes recubren sucesivamente los sedimentos actuales y constituyen posibles rasgos geomorfológicos que pudieran influenciar en las pendientes.

Las pendientes más elevadas fueron verificadas en el sector occidental, siendo la Formación La Popa la única ubicada en el casco urbano de la ciudad de Cartagena. En campo, se determinó una relación sobresaliente entre una pendiente con una elevada inclinación en un salto de falla en la Formación La Popa, corroborando por tanto que la relación pendiente versus tectónica reciente puede ser probada y no es posible renegarla en el área. 
Max Furrier, German Vargas Cuervo. Geomorphology and mapping of the Central Sector of Cartagena de Índias - Colombia

Tomando únicamente a título ilustrativo la legislación brasilera, podría concluirse que gran parte del Cerro La Popa no podría estar ocupado por urbanizaciones, más lo que se verificó en la práctica fue precisamente lo contrario, siendo identificada una ocupación densa y de estrato bajo. Es preciso resaltar que no solo por la inclinación de las pendientes verificadas en las que el cerro se localiza esta población se encuentra en riesgo, si no también, por ser un relieve con muchas fallas verificadas. En efecto, como ya se mencionó, es un relieve producto de una tectónica reciente y por lo tanto, existen aún muchas fallas no verificadas que aumentan el riesgo de la ocupación.

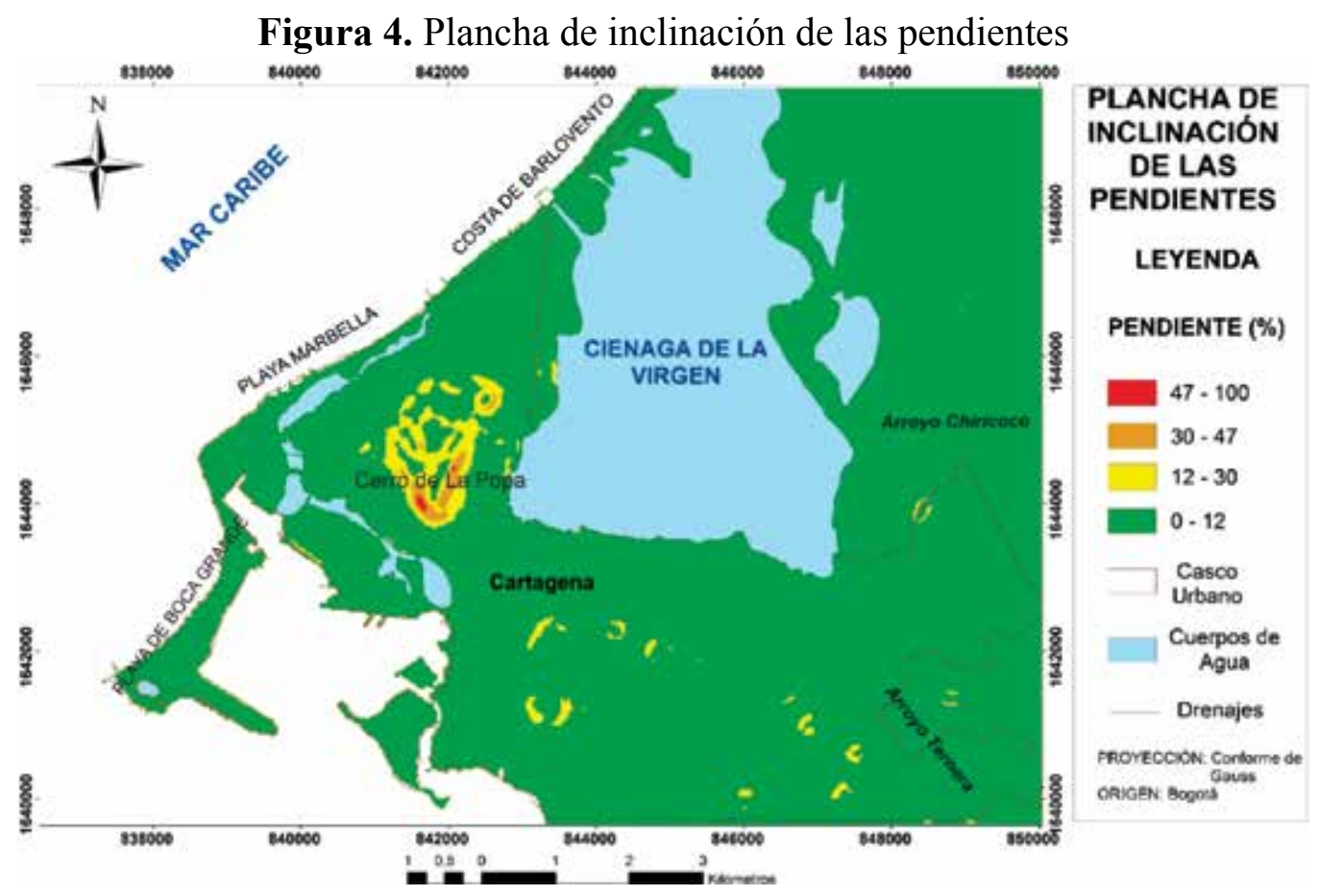

La tabla 3 muestra la proporción en $\mathrm{km}^{2}$ y en porcentaje de cada clase hipsométrica del área de estudio. 
Tabla 3. Clases de inclinación de las pendientes en el área de estudio en $\mathrm{km}^{2} \mathrm{y}$ en porcentaje

\begin{tabular}{|c|c|c|}
\hline Clases de inclinación & Área (km2) & Área (\%) \\
\hline $0-12 \%$ & 86,16 & 98,07 \\
\hline $12-30 \%$ & 1,43 & 1,62 \\
\hline $30-47 \%$ & 0,25 & 0,29 \\
\hline $47-100 \%$ & 0,014 & 0,017 \\
\hline
\end{tabular}

\section{Plancha geomorfológica}

Basado en la construcción y análisis de la plancha geomorfológica (Figura 7), como síntesis de todo este trabajo, fueron identificadas cuatro morfoestructuras para el área de la hoja 23 III D. Estas son; a saber, Cinturón del Sinú, depósitos de terrazas y planicies costeras, depósitos coluvioaluviales y depósitos de playa. Dichas morfoestructuras fueron a su vez subdivididas en varias morfoesculturas de jerarquía menor; a saber, Terrazas y Planicies Costeras, Terrazas y Planicies Coluvioaluviales, Terrazas y Planicies Marinas y Cerros testimonios del Sinú.

Con respecto a los patrones de formas del relieve ( $3^{\text {er }}$ Taxón), en el Cinturón del Sinú, fueron identificadas formas con ápices en mesetas, encontradas en las zonas más bajas de la Formación La Popa y formas con ápices convexos identificados en el Cerro La Popa, mostrando diferentes formas sobre la misma litología.

Los valores encontrados en los tipos de formas del relieve presentan una incisión media de los valles fuerte $(80-160 \mathrm{~m})$ y dimensión interfluvial media grande $(700-1.500 \mathrm{~m})$ para los ápices convexos Dc 42. Estos valores de disección sugieren que la fuerte incisión se da por procesos erosivos agresivos y demuestran que la morfoescultura está en un rápido proceso denudacional, debido a su elevada altimetría y a las altas pendientes.

Una característica del relieve mapeado es la existencia de diferentes morfologías que se desarrollan sobre la Formación La Popa, que tiene forma en ápices convexos (Dc 42) en el Cerro La Popa donde su pendiente es bastante pronunciada frente al continente, y en dirección sureste al cerro formas con ápices en forma de mesetas (Dm 22), donde se sugiere una influencia tectónica más baja porque la distancia entre los dos cerros es pequeña para decir que la diferencia altimétrica fue debido al clima. Es 
Max Furrier, German Vargas Cuervo. Geomorphology and mapping of the Central Sector of Cartagena de Índias - Colombia

visible y notable la influencia tectónica reciente en el desarrollo de esta formación en sus varios afloramientos.

En el relieve agradacional formado por las Terrazas y Planicies Costeras, se presenta un área baja con altitudes máximas de 20 metros al este y al sur de la Ciénaga de La Virgen. La falta de estudios geológicos más detallados impide una descripción geomorfológica precisa. Debido a que la altitud e inclinación de sus pendientes son muy bajas, hoy dichas terrazas y planicies están densamente ocupadas y sufren constantemente por inundaciones.

La referida ocupación se concentra en todo el casco urbano de Cartagena y en las áreas adyacentes, expandiéndose hacia el norte, siguiendo la autopista Cartagena - Barranquilla. Esta circunstancia dificulta sobre manera su correcta delimitación, principalmente, cuando esta morfología se estudia a partir de la categoría Terrazas y Planicies Costeras.

Hay en casi toda la franja costera del casco urbano de Cartagena obras de ingeniería costera para minimizar o impedir la erosión de las playas. No siendo la geomorfología costera el foco central de este trabajo, para una correcta interpretación de esos procesos de erosión costera sería necesario un análisis más profundo sobre el tema. No obstante, lo anterior, cabe resaltar que no es posible concluir que esta erosión costera se debe únicamente a la elevación del nivel medio del mar, pues no existen datos suficientes que permitan llegar a una conclusión de esa naturaleza.

Al respecto, conviene destacar que la principal causa de erosión costera en el mundo es el déficit sedimentario que puede estar relacionado con la ocupación desordenada de la ciudad que atrapan grandes cantidades de sedimentos. Los resultados de este trabajo apuntan a una fuerte tectónica positiva y reciente para la región. Siendo los procesos tectónicos y de erosión costera distintos en la escala temporal, se cree que la tectónica puede afectar el nivel del mar por la tectonoisostasia, ya que no se observa erosión costera en áreas al norte donde no hay fuertes interferencias antrópicas. En áreas con tectónica positiva, la tendencia es de progradación en la línea de costa y no de erosión como se verificó en todo el casco urbano de Cartagena (Figura 5). 
Figura 5. Imagen de Google Earth georreferenciada de la zona de Boca Grande, donde es posible visualizar las obras de ingeniería para la contención de la erosión costera.

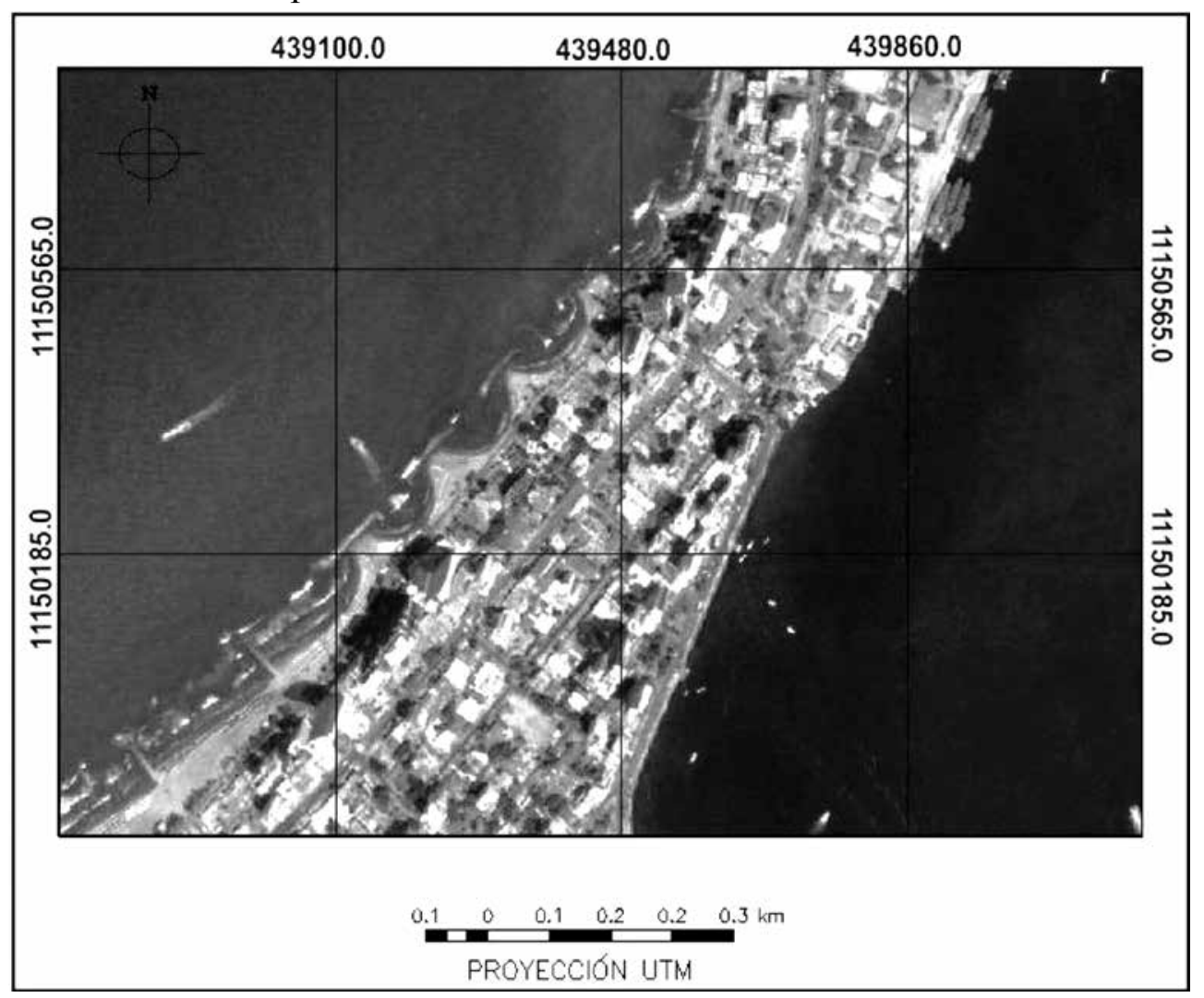

Fuente: Imagen satelital de 01/04/2015.

Siendo la ciudad más turística de Colombia, la cuestión de la erosión costera en el casco urbano requiere estudios intensos y detallados para intentar determinar su causa principal y así buscar minimizar los efectos negativos e intensos de este proceso. En la zona de Boca Grande (Figura 6), además de encontrarse grandes redes hoteleras, edificios lujosos y las principales playas del casco urbano, se concentra el metro cuadrado más caro de Cartagena y unos de los más costosos de toda Colombia. No obstante, lo anterior, la playa está sufriendo un intenso proceso erosivo solamente contenido por obras de ingeniería. 
Max Furrier, German Vargas Cuervo. Geomorphology and mapping of the Central Sector of Cartagena de Índias - Colombia

Estas obras son compuestas por quiebra-mares y molles, todos hechos con pedazos de rocas calizas de la Formación La Popa. Además de los efectos en el paisaje no ser atractivos desde el punto de vista turístico, las rocas calizas, principalmente porosas y de baja densidad como son las rocas de la Formación la Popa, no son adecuadas para este tipo de obra. Por lo tanto, no solo Cartagena necesita de más estudios sobre los procesos erosivos de sus playas, como también necesita de una planificación más adecuada para la contención de este proceso.

Figura 6. Fotografía mostrando las obras de ingeniería al largo de toda Playa de Boca Grande hechas con rocas calizas de la Formación La Popa.

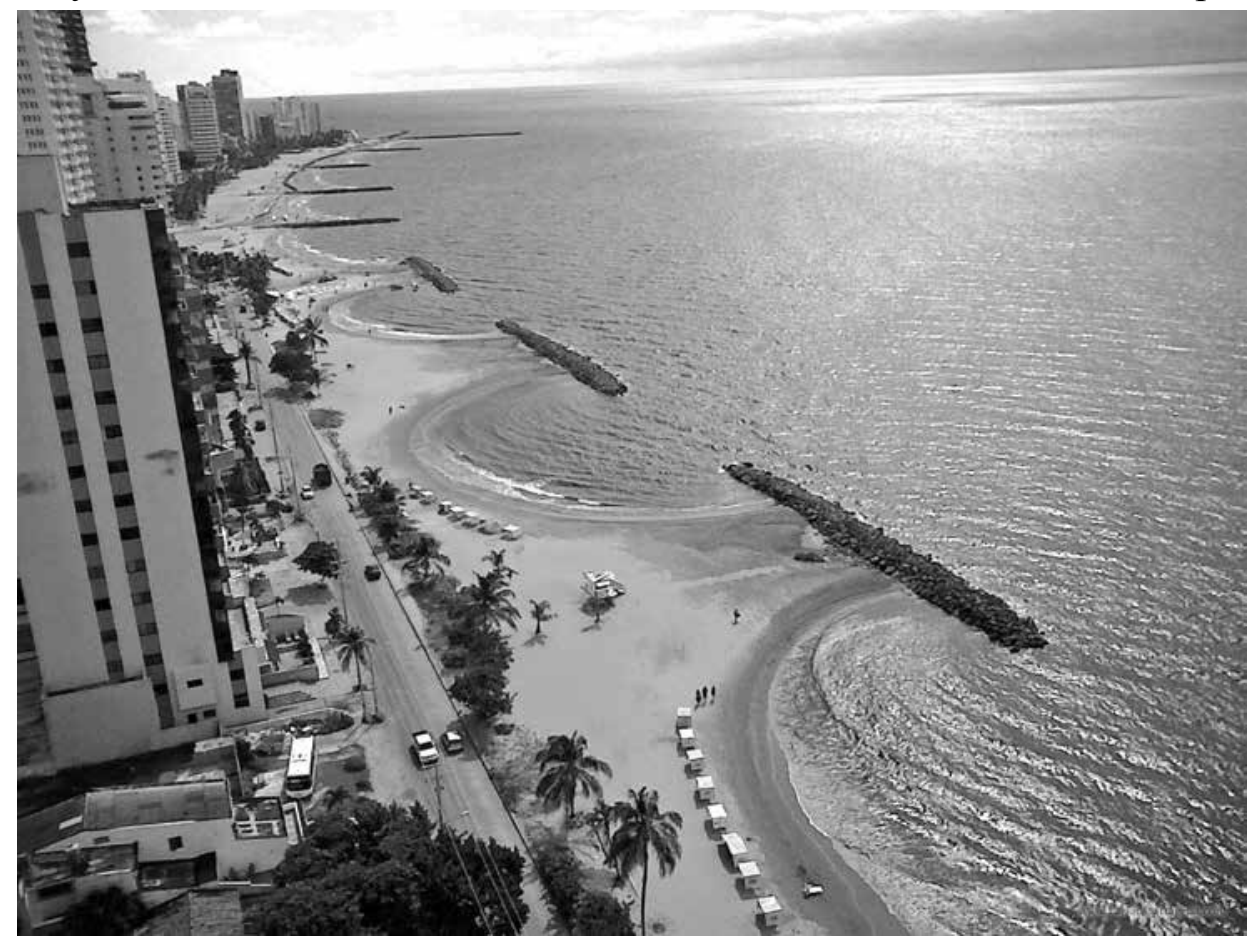

Fuente: www.paisoncartagena.com, obtenida en 04/05/2016. 


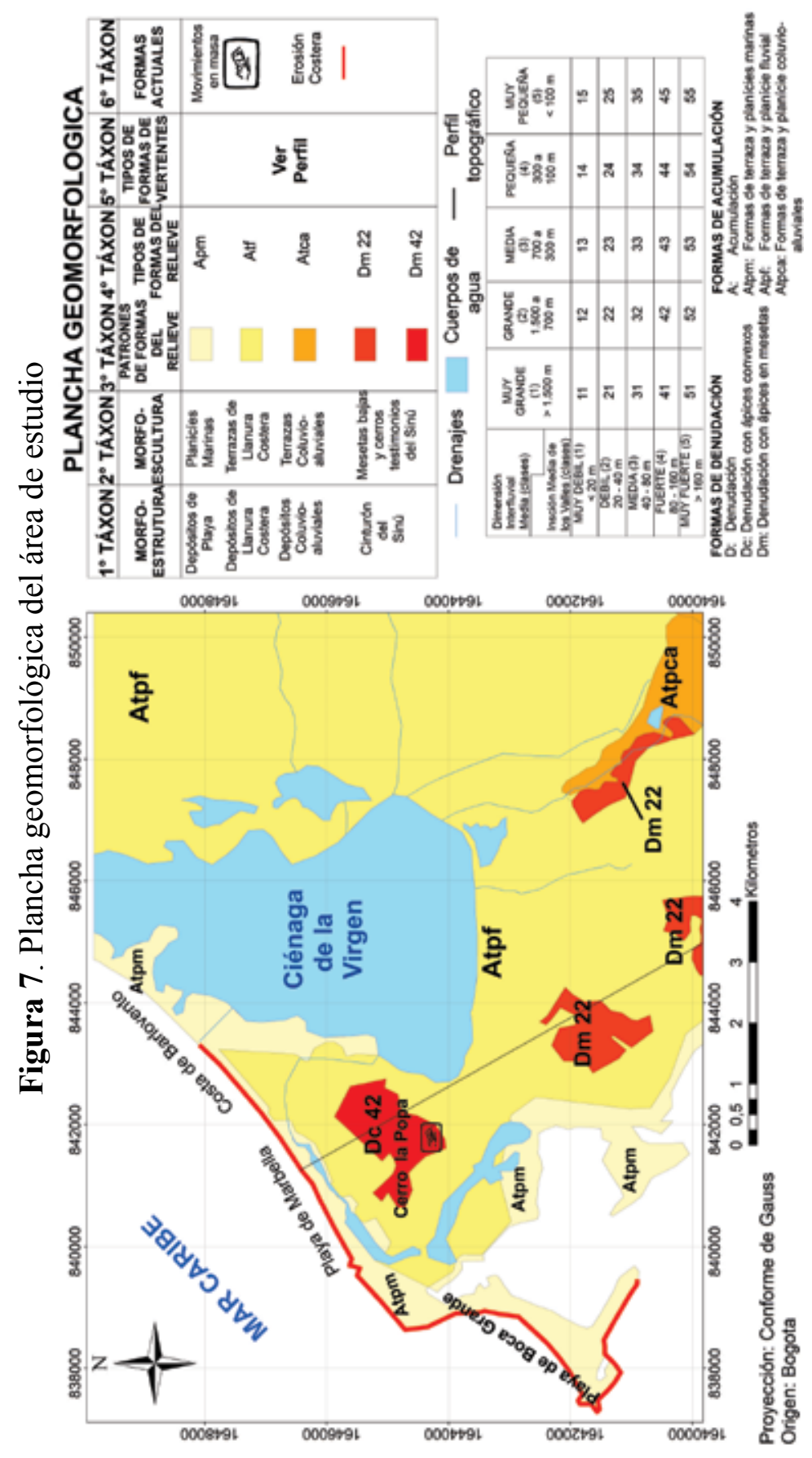


El quinto taxón se refiere a los sectores de las pendientes, que serán descritos en una sección específica en este artículo. El sexto taxón corresponde a las formas del relieve resultantes de procesos actuales, naturales o antrópicos. En el área de estudio se pueden encontrar principalmente, formas hechas por la erosión costera y formas resultantes de movimientos de masa.

\section{Quinto Taxón - Descripción de las pendientes}

La descripción de las formas de las pendientes corresponde al $5^{\text {to }}$ taxón de la metodología de cartografía geomorfológica empleada en esta investigación, equivale a la descripción de las formas de las pendientes identificadas en una sección transversal trazada en el área de estudio.

Las formas de las pendientes (Figura 8) son identificadas por medio de un perfil topográfico en dirección SW-NE elegido por abarcar las principales formaciones geológicas-geomorfológicas del área. Fueron identificadas pendientes rectilíneas, convexas y convexo-rectilíneas, debido al perfil estar trazado sobre las formaciones La Popa y Bayunca que se encuentran dentro del casco urbano de Cartagena.

Las pendientes más heterogéneas son verificadas en el Cerro de La Popa donde las pendientes con dirección SE son rectilíneas y con la declividad mucho más acentuada que las encontradas al Noreste que son rectilíneas y convexas con declividades mucho más bajas (Figura 8). 

de Cartagena de Indias - Colombia

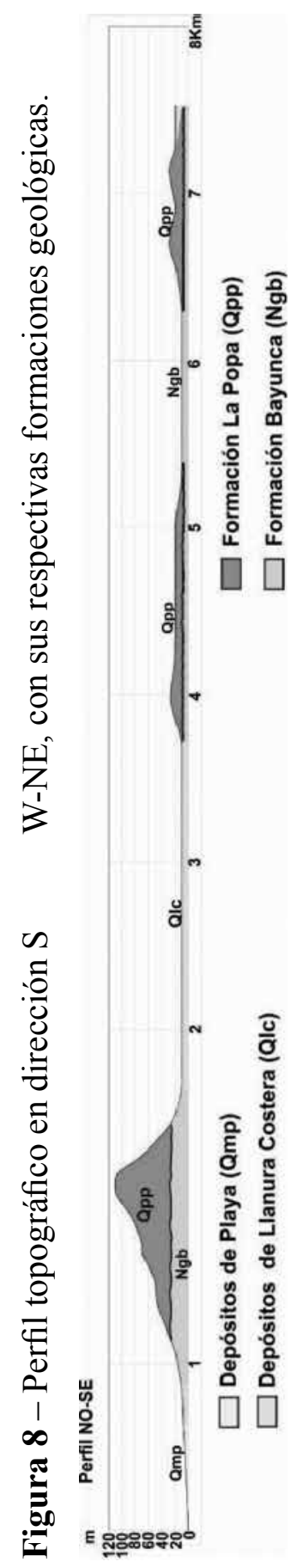


Max Furrier, German Vargas Cuervo. Geomorphology and mapping of the Central Sector of Cartagena de Índias - Colombia

\section{Discusión de resultados}

Esta investigación permitió realizar un diagnóstico geológico y geomorfológico del sector central de la ciudad de Cartagena de Indias, por medio de la metodología de cartografía geomorfológica desarrollada por Ross (1992) ampliamente utilizada en Brasil, inclusive en el mapeo geomorfológico de dos importantes estados brasileños: São Paulo y Paraná.

La ciudad de Cartagena, aunque se encuentra predominantemente en terrenos planos, donde presenta una fuerte expansión urbana, industrial y portuaria, también posee relieve con formas escarpadas, donde la tectónica actuó fuertemente tanto en su formación como en su evolución a través de un sin número de fallas verificadas por donde las aguas pluviales se filtran. Por lo tanto, las fallas actúan de forma intensa en la esculturación del relieve actual pudiendo aumentar significativamente el riesgo para la población que se localiza alrededor.

El hecho de que en Colombia la Geomorfología esté basada solamente en procesos exógenos puede resultar en una falta de datos que lleve a equívocos peligrosos, pues gran parte de ese país se encuentra en zonas activas, tal y como ocurre con el área objeto de estudio. En ese sentido, es claro que el basamento rocoso, y sus rasgos geomorfológicos como fallas y pliegues no pueden ser renegados en los estudios y mapeos geomorfológicos, así también como la acción humana, fuertemente verificada en la zona costera.

Por lo anterior, es de vital importancia la ampliación de este estudio en una escala que permita un mayor detalle y también una mayor cobertura, para de esta forma, utilizar los datos físicos cualitativos y cuantitativos aquí presentados. Un análisis de estos datos en forma conjunta con informaciones socio-económicas, permitirá optimizar el uso y ocupación del territorio aplicando metodologías adecuadas para una planificación ambiental y de ordenamiento territorial de la ciudad y de sus alrededores. Esta es la propuesta central de este trabajo; es decir, presentar una metodología de mapeo geomorfológico y temático sólidamente sustentado en la cuantificación, morfometría y basamento rocoso, produciendo así un mapa/plancha síntesis del estudio general, con el propósito de ser una herramienta esencial a futuros trabajos de planeamiento y ordenamiento territorial.

Sin contar con los datos del medio físico ya mencionados, no es posible llevar a cabo, ningún tipo de planificación ambiental o de ordenamiento territorial. Un detallado conocimiento del relieve y su basamento rocoso, 
tanto en sus aspectos estructurales como esculturales, resulta fundamental por el mismo ser el escenario de las diferentes actividades económicas y sociales del ser humano. El hombre desarrolla todas sus actividades sobre el relieve y es por ese motivo que la Geomorfología constituye una pieza de gran importancia dentro de los estudios geográficos.

\section{Agradecimientos}

Los autores de este artículo agradecen al Conselho Nacional de Desenvolvimento Cientifico e Tecnológico - CNPq a través del proceso 470200/2013-8 por el apoyo financiero.

\section{Referencias}

Ángel, C., Esquivel, J. y Sarmiento, G. (1985). Geología del Departamento de Bolivar al norte del Canal del Dique. Bogotá: IGEOMINAS. (143 p.) (Informe, 1941).

Barbosa, G. V., Silva, T. C., Natali Filho, T., Del'Arco, D. M. y Costa, R. C. R. (1984). Evolução da metodologia para mapeamento geomorfológico do projeto RADAMBRASIL. In: Projeto RADAMBRASIL. Boletim Técnico. Salvador, MME. Série Geomorfologia, 1(187), 3-26.

Carvajal, J. H. (2012). Propuesta de estandarización de la cartografía geomorfológica en Colombia. Servicio Geológico Colombiano. (83 p.)

INGEOMINAS, Instituto Colombiano de Geología y Minería. (2004). Geología de los cinturones del Sinú, San Jacinto y borde occidental del valle inferior del Magdalena - Caribe Colombiano, escala: 1:300.000. Bogotá: INGEOMINAS. (134 p.)

IGAC, Instituto Geográfico Agustín Codazzi. (1996). Hoja N. 23 III D. Bogotá: IGAC, 1 hoja, B/N. $90 \mathrm{~cm}$ x $70 \mathrm{~cm}$. Escala 1:25.000.

Feitosa, C. A. (1996). Dinâmica dos processos geomorfológicos da área costeira a nordeste da ilha do Maranhão. Tese Doutorado. Instituto de Geociências e Ciências Exatas, Universidade Estadual Paulista. Brasil. (249 p.)

Furrier, M. (2007). Caracterização geomorfológica e do meio físico da folha João Pessoa 1:100.000. Tese Doutorado. Faculdade de Filosofia, Letras e Ciências Humanas (FFLCH), Universidade de São Paulo. Brasil. (213 p.) 
Max Furrier, German Vargas Cuervo. Geomorphology and mapping of the Central Sector of Cartagena de Índias - Colombia

Herz, R. y De Biasi, M. (1989). Critérios e legendas para macrozoneamento costeiro. Ministério da Marinha/Comissão Interministerial para os Recursos do Mar. Brasília. (198 p.)

Mescerjakov, J. P. (1968). Les concept de morphostruture et de morphosculture: un nouvel instrument de l'analyse géomorphologique. Annales de Géographie, 77 (423), 539-552.

Ross, J. L. (1992). O registro dos fatos geomórficos e a questão da taxonomia do relevo. Revista do Departamento de Geografia da USP, 6 (1), 17-29. 